\section{The progression of severe aplastic anemia to hypoplastic leukemia in a long-term obser- vation after the administration of pegylated rHuMGDF}

\author{
Maho Ishikawa, Akira Matsuda, \\ Daisuke Okamura, Tomoya Maeda, \\ Nobutaka Kawai, Norio Asou, Masami \\ Bessho \\ Department of Hemato-Oncology, \\ Saitama Medical University \\ International Medical Center, Hidaka, \\ Saitama, Japan
}

\begin{abstract}
Thrombopoietin (TPO) is a critical regulator of hematopoiesis. We previously reported that a severe aplastic anemia (SAA) who received a short-term administration of pegylated recombinant human megakaryocyte growth and development factor (rHuMGDF). A trilineage hematologic response was induced, however the patient was diagnosed with leukemia after nine years and eight months from administration of rHuMGDF. In recent reports, somatic mutations in myeloid cancer candidate genes were present in one-third of the AA. A mutant clone may be expanded by rHuMGDF in our patient. The long-term safety of patients treated with TPO and eltrombopag remains unknown. Careful observations are warranted hereafter.
\end{abstract}

\section{Introduction}

Aplastic anemia (AA) is one of the lifethreatening bone-marrow failure syndromes characterized by pancytopenia and hypoplastic marrow. The appropriate treatments for severe AA (SAA) including immunosuppressive therapy (IST) and hematopoietic stem cell transplantation (SCT) are selected mainly depending on age and stage of severity. Although longterm survival can be expected, management of SAA requires careful attention to clonal evolution.

We report here a patient with a hypoplastic leukemia evolved from SAA. A short-term administration of rHuMGDF is suspected of contributing to this clonal evolution. A decade of intriguing clinical course of our patient is consistent with recent findings in clonal hematopoiesis in AA. The addition of eltrombopag to IST has received attention due to therapeutic effect in AA. We report here our case to promote awareness of long-term observation in AA who especially intend to treat with eltromlbopag.

\section{Case Report}

A 46-year-old Japanese male was diagnosed with SAA in February 1997. Laboratory tests showed a hemoglobin of $4.0 \mathrm{~g} / \mathrm{dL}$, platelet count of $0.4 \times 10^{9} / \mathrm{L}$, leukocyte count of $3.0 \times 10^{9} / \mathrm{L}$, and neutrophil count of $0.45 \times 10^{9} / \mathrm{L}$ on his initial visit. He was treated with a combination therapy of cyclosporine A (CyA) and recombinant human granulocyte colonystimulating factor (rhG-CSF), and he was referred to our hospital seven months after diagnosis. He refused allogeneic stem cell transplantation and was thus treated with a combination therapy of horse anti-thymocyte globulin (ATG), CyA and rhG-CSF. The changes of laboratory tests before and three months after treatment of IST were limited. Increase in hemoglobin level from $4.0 \mathrm{~g} / \mathrm{dL}$ to $7.6 \mathrm{~g} / \mathrm{dL}$, platelet count from 4.0 $\mathrm{x} 10^{9} / \mathrm{L}$ to $30 \times 10^{9} / \mathrm{L}$, and neutrophil count from $0.45 \times 10^{9} / \mathrm{L}$ to $1.3 \times 10^{9} / \mathrm{L}$. Once a minimal response had been obtained, however, cytopenia gradually got worsened in one year. Therefore, he participated in a clinical trial of phase I/II rHuMGDF by daily intravenous injection for 14 days. He showed trilineage response, especially in the erythroid line, at 6-16 weeks from the start of treatment. The early effect had been transient. From at six months after start of rHuMGDF treatment, he gradually showed a continued trilineage response, particularly in the erythroid line. His hemoglobin level increased to more than $10 \mathrm{~g} / \mathrm{dL}$ without additional transfusions or treatments, neutrophil count was more than $2.0 \times 10^{9} / \mathrm{L}$ and platelet count of $3.0 \times 10^{9} / \mathrm{L}$. No adverse events or neutralizing antibodies were observed. ${ }^{1}$ Stable cell counts were maintained for more than six and a half years. Thereafter, at his request, he did not consult our hospital.

The patient was readmitted to our hospital with signs of anemia at nine years and eight months after the cessation of rHuMGDF. Laboratory tests showed a hemoglobin level of $5.7 \mathrm{~g} / \mathrm{dL}$, platelet count of $13 \times 10^{9} / \mathrm{L}$, and leukocyte count of $1.95 \times$ $10^{9} / \mathrm{L}$ without blasts. His bone marrow (BM) showed hypocellularity with 23\% blasts, which were peroxidase $(+), \mathrm{CD} 13$ $(+)$, CD33 (+), CD56 (+), and CD117 (+) (Figure 1). A cytogenetic analysis of BM cells revealed 47, XY, +8 [4] / 46, XY [16]. The patient was diagnosed with hypoplastic leukemia. He was treated with low-dose cytarabine and aclarubicin in combination
Correspondence: Maho Ishikawa, Department of Hemato-Oncology, Saitama Medical University International Medical Center, 1397-1 Yamane, Hidaka, Saitama, 350-1298, Japan.

Tel.: +81.42.984.4111 - Fax: +81.42.984.4567.

E-mail: miskw@saitama-med.ac.jp

Key words: Aplastic anemia; acute myeloid leukemia; thrombopoietin; clonal evolution.

Acknowledgments: the authors would like to thank A. Matsuda for useful discussions. Finally, we are grateful to the referees for useful comments.

Conflicts of interest: Akira Matsuda received honoraria from Kyowa Hakko Kirin Co., Ltd., and GlaxoSmithKline K.K. The remaining authors declare no conflicts of interest.

Received for publication: 13 March 2018.

Accepted for publication: 24 June 2018.

This work is licensed under a Creative Commons Attribution-NonCommercial 4.0 International License (CC BY-NC 4.0).

CC Copyright M. Ishikawa et al., 2018

Licensee PAGEPress, Italy

Hematology Reports 2018; 10:7679

doi:10.4081/hr.2018.7679

with G-CSF (CAG regimen). After one course of the $\mathrm{CAG}$ regimen, BM blasts decreased to less than $0.9 \%$, but with the persistence of pancytopenia and transfusion dependency. Difficulties were associated with continuing subsequent chemotherapy due to invasive pulmonary aspergillosis. He died of disease progression 18 months after the diagnosis of hypoplastic leukemia.

\section{Discussion and Conclusions}

We report here the meaningful clinical course of a Japanese patient with SAA, whose condition progressed to hypoplastic leukemia in a long-term observation after the administration of rHuMGDF following our previous report. In our knowledge, our case is the first acute myeloid leukemia (AML) patient in subjects who ever received TPO. The clinical development of TPO was canceled principally because the production of the anti-TPO antibody in subjects received TPO. The long-term followup of these subjects is not done. Therefore, there is no knowing about their clinical course. Instead of TPO, TPO receptor agonist was developed thereafter.

TPO is a critical regulator of hematopoiesis. The TPO receptor $c-m p l$ is 
expressed on hematopoietic stem cells (HSCs), and knockout mice of the $c-m p l$ lack HSCs. Therefore, we thought that the short-term administration of rHuMGDF became a trigger for the expansion of normal HSCs in our AA patient. Eltrombopag is a TPO receptor agonist that is used to treat patients with idiopathic thrombocytopenic purpura. It was recently shown to be effective for AA patients. In SAA patients with persistent pancytopenia after IST, hematologic responses were observed with the administration of eltrombopag. Desmond et al. reported that a treatment with eltrombopag induced tri- and bilineage responses as well as the maintenance of normalized hematopoiesis after treatment cessation. $^{2}$ These findings indicate that eltrombopag promotes hematopoiesis in AA patients by stimulating stem and progenitor cells. This effect of eltrombopag may be similar to that of rHuMGDF in our patient. In the study conducted by Desmond et al., 8 patients, including 6 nonresponders and 2 responders, developed new cytogenetic abnormalities when treated with eltrombopag, including 5 with the loss or partial deletion of chromosome 7 . None of these patients progressed to AML, although 5 out of 8 underwent allogeneic SCT. Our SAA patient progressed to leukemia in the longterm observation. In AA patients, there is no knowledge on the risk of clonal evolution in long-term follow-up period of eltrombopag. The comparison between clonal evolution cases in National Institutes of Health (NIH) reported by Desmond et al. and our case is shown in Table 1. The median follow-up period from eltrombopag administration start to clonal evolution is only three months, and is shorter than our case. If the follow-up period becomes longer, the number of patients who develop clonal evolution with eltrombopag may further increase. That is the reason we emphasize the necessity of long-term observation after treatment with eltrombopag through our case.

Naturally, SAA patients have a risk of clonal evolution. A recent study showed that somatic mutations in myeloid cancer candidate genes were present in one-third of AA patients, and mutations in a subgroup of genes that included DNMT3A and ASXL1 were associated with poorer outcomes. ${ }^{3}$ Also, in AA, a concept of clonal hematopoiesis has received considerable attention adopt a perspective of clonal evolution in recent years. Moreover, clonal hematopoiesis with somatic mutations has been observed in at least $10 \%$ of individuals older than 65 years of age. Detectable clonal expansion most frequently involves somatic mutations in DNMT3A, ASXL1, and TET2, which have previously been implicated in hematologic cancers. ${ }^{4}$ Steensma et al. proposed the term clonal hematopoiesis of indeterminate potential (CHIP) to describe individuals with a hematologic malignancy-associated somatic mutation in blood or BM, but without other diagnostic criteria of a hematologic malignancy. ${ }^{5}$ CHIP are seen in elderly and clonal evolution to myelodysplastic syndromes (MDS)/AML may occur around $1 \%$ per year. However, in CHIP, the risk of clonal evolution may be increased by administration of TPO receptor agonist. About $15 \%$ of AA patients have a risk of development of MDS/AML. Recent study has revealed that clonal hematopoiesis is prevalent in AA with somatic gene mutations and progress to MDS/AML at a high rate. In a subgroup of AA, patients already have somatic mutations at the onset of $\mathrm{AA}$, and they progress to MDS/AML is surmised.

We think that a short-term administration of rHuMGDF led to his clonal evolution. However, the proposed scenario has not been proven. An association with administration of G-CSF is worth considering. It is reported that long-term use of GCSF is related to development of MDS and AML. ${ }^{6}$ On the other hand, certain data also suggest that there is no correlation between G-CSF and the risk of developing MDS/AML. ${ }^{7,8}$ It remains a controversial subject. In our case, the combination thera-

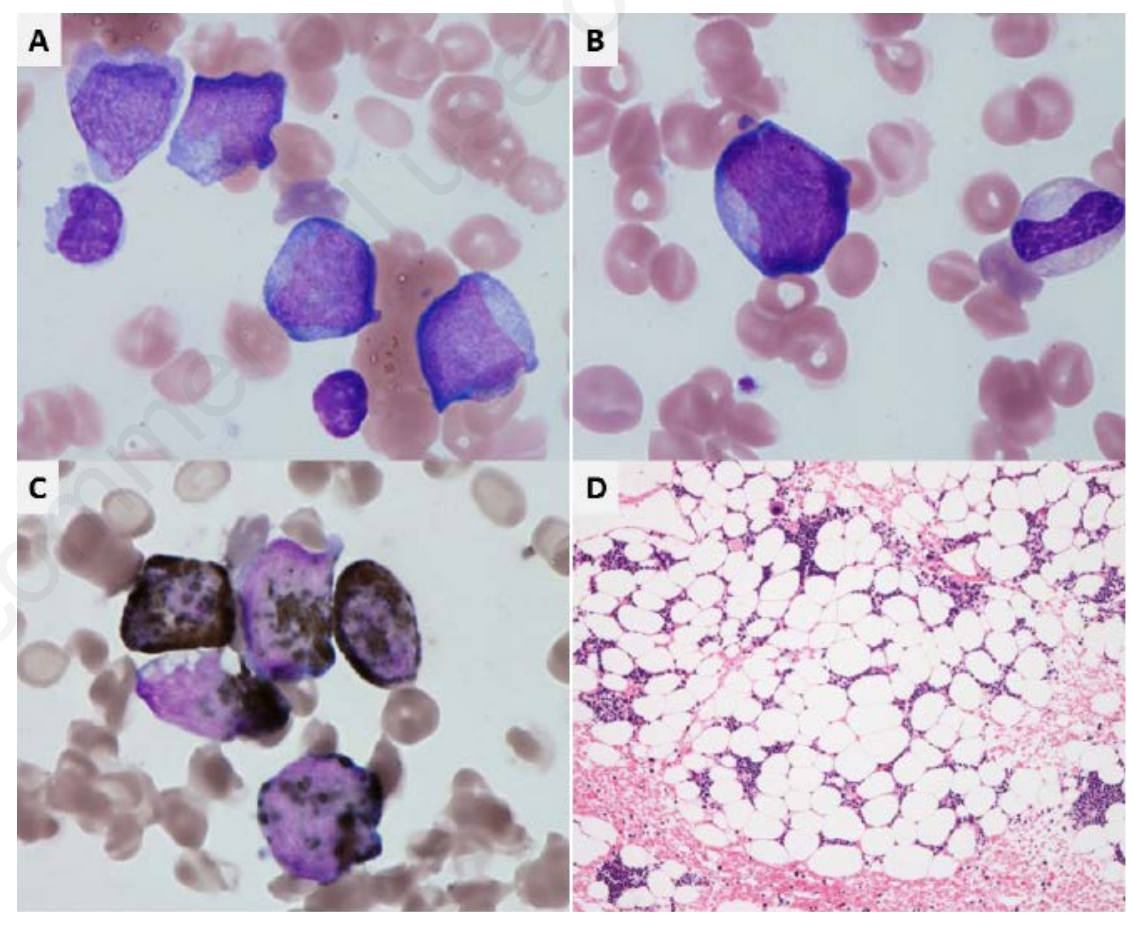

Figure 1. Morphology of bone marrow at the diagnosis as hypoplastic leukemia. A) and B) May-Giemsa stain. Blasts were slightly small, had Auer body and no dysplasia. C) Peroxidase stain. Blasts were positive for peroxidase stain, CD13, CD33, CD56, and CD117. D) Bone marrow clot section showed approximately $20 \%$ cellularity.

Table 1. Comparison between NIH cases and our case.

\begin{tabular}{lc} 
& NIH cases (N=8) \\
Karyotype at clonal evolution, $n(\%)$ & Poor*, $5(62.5 \%)$ \\
Period of administration of eltrombopag, months (range) & $3(3-13)$ \\
\hline Efficacy of eltrombopag (response rate), n (\%) & $2(25.0 \%)$ \\
Period from eltrombopag administration start to clonal evolution, months (range) 8 & $3(3-13)$
\end{tabular}


py of ATG, CyA and rhG-CSF achieved only limited hematological response. The changes of laboratory values between before IST and 3 months after IST were slight. On the other hand, an obvious trilineage response was observed by short-term administration of rHuMGDF. Although early response was transient, response over at least 6 and a half years was seen continuously. It implicated that the administration of rHuMDGF was more relevant as possible inducer of cell proliferation than G-CSF. We assume that our patient might have some mutations at the onset of AA and a continued trilineage response after start of rHuMGDF treatment was maintained by clonal hematopoiesis associated with somatic mutations. This clonal hematopoiesis may be similar to hypoplastic MDS. Unfortunately, there was no such knowledge at that time, we could not analyze about gene mutations of our patient by molecular biological technique. We now think the analysis of gene mutations should be desirable before TPO receptor agonist treatment in AA.

We report here a patient with a hypoplastic leukemia evolved from SAA. A short-term administration of rHuMGDF is suspected of contributing to this clonal evolution. We observed about a decade of clinical course in our AA patient treated with
rHuMGDF. If similar patients exist, longterm follow-up of subjects who ever received TPO may be necessary. Frequency of somatic mutations in AA patients is higher than that in healthy subjects. Therefore, in AA patients, eltrombopag may be more likely to derive clonal evolution. The longterm safety of eltrombopag remains unknown. We think our case gives proper notice for hematologists about needs of careful and over the long-term observation in AA who especially intend to treat with eltrombopag.

\section{References}

1. Matsuda A, Misumi M, Ishikawa M, et al. Long-term improvement of anaemia in a patient with aplastic anaemia by short-term administration of pegylated recombinant human megakaryocyte growth and evelopment factor. $\mathrm{Br} \mathrm{J}$ Haematol 2004;125:818-89. Erratum in Br J Haematol 2004;126:284-5.

2. Desmond R, Townsley DM, Dumitriu B et al. Eltrombopag restores trilineage hematopoiesis in refractory severe aplastic anemia that can be sustained on discontinuation of drug. Blood 2014; 123:1818-25.

3. Yoshizato T, Dumitriu B, Hosokawa K, et al. Somatic mutations and clonal hematopoiesis in aplastic anemia. N Engl J Med 2015;373:35-47.

4. Genovese G, Kähler AK, Handsaker RE, et al. Clonal hematopoiesis and bloodcancer risk inferred from blood DNA sequence. N Engl J Med 2014;371:2477-87.

5. Steensma DP, Bejar R, Jaiswal S, et al. Clonal hematopoiesis of indeterminate potential and its distinction from myelodysplastic syndromes. Blood 2015;126:9-16.

6. Kojima S, Ohara A, Tsuchida M, et al. Risk factors for evolution of acquired aplastic anemia into myelodysplastic syndrome and acute myeloid leukemia after immunosuppressive therapy in children. Blood 2002;100:786-90.

7. Imashuku S, Hibi S, Bessho F, et al. Detection of myelodysplastic syndrome/acute myeloid leukemia evolving from aplastic anemia in children, treated with recombinant human GCSF. Hematologica 2003;88:e136-41.

8. Locasciulli A, Arcese W, Locatelli F, et al. Treatment of aplastic anaemia with granulocyte-colony stimulation factor and risk of malignancy. Italian Aplastic Anaemia Study Group. Lancet 2001;357:43-4. 